\title{
Permanencia estudiantil en programas de posgrado e-learning: un caso de estudio
}

\author{
Rosa E. Quintero-Guasca, Maribel Avellaneda-Nieves, Mónica Cristancho-García, e Irlesa I. Sánchez-Medina \\ Centro de Educación Virtual CVUDES, Universidad de Santander, Colombia. (correo-e: rosa.quintero@cvudes.edu.co; \\ maribel.avellaneda@cvudes.edu.co; monica.cristancho@udes.edu.co; irlesa.sanchez@cvudes.edu.co)
}

Recibido Sep. 25, 2020; Aceptado Nov. 24, 2020; Versión final Ene. 22, 2021, Publicado Jun. 2021

\begin{abstract}
Resumen
El objetivo principal de esta investigación es examinar los factores que inciden en la permanencia estudiantil en un programa académico de posgrado en metodología virtual de una universidad colombiana. A través de revisión de literatura en programas de educación superior y el desarrollo de un ejercicio de percepción aplicado a estudiantes, se determinan situaciones que afectan la continuidad académica y se generan estrategias para el fomento de la permanencia y graduación de estudiantes. Los resultados muestran que los factores con mayor incidencia en la permanencia estudiantil son de carácter individual, socioeconómico y académico, mientras que los factores tecnológicos e institucionales se consideran de menor relevancia. Se concluye que en tanto las motivaciones que conllevan a los estudiantes a abandonar sus estudios son diversas y complejas, se precisan estrategias que aborden la permanencia desde diferentes dimensiones y fortalezcan la institución en el análisis de escenarios internos y externos para mejorar la calidad educativa y la finalización exitosa de la formación.
\end{abstract}

Palabras clave: permanencia estudiantil; educación; estudios de posgrado; virtual; e-learning

\section{Student permanence in e-learning postgraduate programs: a case study}

\begin{abstract}
The primary objective of this research study is to examine the factors that affect student permanence in a virtual methodology postgraduate academic program at a Colombian university. A literature review on student permanence in higher education programs is performed and a perception exercise is developed and applied to students. The situations that affect academic continuity are determined and strategies are generated to promote student permanence and graduation. The results show that the factors that have the greatest impact on student permanence are personal, socioeconomic, and academic, while technological and institutional factors are less relevant. It is concluded that the motivations that lead students to drop out of their studies are diverse and complex and that different strategies are needed to increase student permanence. This includes strengthening the capabilities of educational institutions to analyze internal and external scenarios to improve education quality and successful completion of academic programs.
\end{abstract}

Keywords: student permanence; education; graduate studies; virtual; e-learning 


\section{INTRODUCCIÓN}

La permanencia estudiantil es un tema relevante que se ha abordado desde diferentes ópticas. En Colombia por ejemplo, durante un tiempo el Ministerio de Educación Nacional (MEN) habló de deserción y no de permanencia, enfocándose más en el problema que en la prevención, desde una perspectiva de cifras y estadísticas que no abordaba los aspectos de fondo de la situación. Actualmente esta mirada ha cambiado y la permanencia estudiantil se considera un fenómeno explicado por múltiples causas en el que intervienen diferentes actores, con impacto no solo económico sino también en el proyecto de vida del estudiante y su familia. En ese sentido, el MEN promueve estrategias que fortalecen la capacidad de las Instituciones de Educación Superior (IES) para abordar la permanencia y graduación estudiantil, articuladas con políticas que mejoran la calidad en procesos educativos con la intención de que los estudiantes continúen el sistema de educación superior (Parody et al., 2015). En una revisión que incluyó documentos institucionales del MEN y dieciocho universidades del país, Suárez y Díaz (2015) destacan las acciones adelantadas por las IES en el marco de "programas de fomento de la permanencia y el éxito académico" desde cuatro dimensiones diferentes y complementarias: social, psicopedagógica, política y educativa-cultural.

Barragan y González (2017), a partir del trabajo de Tinto (1975) considerado un aporte clásico para quienes investigan en deserción y permanencia universitaria, indican que el modelo de interacción propuesto por Tinto permite al alumno alternar el estudio con su familia, trabajo y sociedad para la integración positiva con pares, docentes, actividades extracurriculares, metas de graduarse y responsabilidades con la institución, destacando el compromiso del estudiante como elemento fundamental para garantizar la permanencia. Basados también en el modelo de Tinto, Urbina y Ovalles (2016) dan a conocer factores que inciden en la permanencia: motivación para la integración académica y social del estudiante, esfuerzo y deseo de superación personal, trabajo colaborativo, buenas prácticas pedagógicas y relaciones humanas.

A través del análisis de la integración académica y social a la vida universitaria, Rueda et al. (2020) determinan un perfil de riesgo de deserción que destaca condiciones de desadaptación al contexto universitario, pertenencia a familias monoparentales y disfunción familiar, frente a lo cual recomiendan programas de acompañamiento a estudiantes en aspectos socioeconómicos, psicológicos, institucionales y académicos. Por su parte, Castro et al. (2020) exploran la relación entre los factores de ingreso de los estudiantes con el resultado final (graduación, deserción o rezago) destacando la importancia de los estudios de trayectoria que abarcan todo el ciclo académico, lo que impone grandes retos a las instituciones educativas dada la exigencia de largos períodos de seguimiento en los que se requiere contar con adecuados sistemas de información.

En otros países, las investigaciones también evidencian interés sobre la permanencia estudiantil a partir del análisis de los factores que la definen e influencian y el desarrollo de estrategias para su fomento. El sistema educativo de Brasil genera políticas de ingreso a la universidad con el propósito de apoyar a los estudiantes (Piva y De Moraes, 2015) y trabaja en el monitoreo de profesores a estudiantes en el entorno de aprendizaje desde las dimensiones pedagógica, administrativa e institucional como estrategias para afianzar la permanencia estudiantil (Oliveira et al., 2017). En Chile, Von Hippel y Hofflinger (2017) analizan a estudiantes en riesgo de abandono poniendo en evidencia bajo control de políticas, poca ayuda financiera e ineficacia de los programas de éxito estudiantil implementados en algunas universidades del país. Por su parte, Huerta y Gaete (2019) exploran el caso de universidades chilenas a través del análisis de convenios marco que fortalecen la permanencia estudiantil como parte del mejoramiento de la calidad en las IES. Estudios realizados en Argentina, identifican los atributos que caracterizan los casos de deserción y su relación con el desempeño académico utilizando técnicas de clasificación en minería de datos (Eckert y Suénaga, 2015), y promueven la implementación de instructivos en beneficio de la permanencia, fortaleciendo la construcción de un aprendizaje con sentido, tareas dinámicas participativas, procesos de interacción, autonomía, y orientaciones para autorregular recursos de aprendizaje (Paoloni et al., 2015).

Una investigación adelantada en Australia por Carroll et al. (2013), sugiere una variedad de factores situacionales, disposicionales y actitudinales que impactan la permanencia de los estudiantes de posgrado en un contexto determinado y proponen estrategias orientadas a favorecer la continuidad y finalización del proceso de formación. En Letonia, Paura y Arhipova (2014) partiendo de la importancia de reconocer las razones del abandono estudiantil para entender el fenómeno y contribuir a la generación de alternativas para su abordaje, identifican los bajos niveles académicos de los estudiantes desde sus bases en la educación secundaria y la poca motivación para estudiar.

De manera particular, la permanencia estudiantil en programas e-learning plantea desafíos tanto en la definición como en la forma de abordar el tema, supeditado al contexto de sus actores, dándole a cada institución la responsabilidad de hacer su propio análisis basado en sus necesidades y oportunidades. La evolución de los recursos tecnológicos ha permitido incursionar en el e-learning como alternativa de empoderamiento de los procesos de enseñanza-aprendizaje con nuevos contextos educativos y recursos 
para el desarrollo exitoso de cualquier programa académico, considerando la actitud del usuario final (estudiantes) hacia la aplicación de la tecnología de la información como factor primordial (Popovici y Mironov, 2015). El e-learning como método de aprendizaje, inicia conectando la web al recurso de información, y luego se extiende a un enfoque pedagógico para la gestión del aprendizaje combinado, lo que genera resultados importantes con ventajas para ser utilizado de manera efectiva en la educación a distancia y presencial mediante gestores de aprendizaje (Hubackova, 2015).

Instituciones de educación superior en línea / a distancia como la Universitat Oberta de Catalunya (UOC), requieren una definición de abandono que refleje la naturaleza de la educación para estudiantes adultos con compromisos laborales y familiares que tienden a tomar descansos académicos. El abandono definitivo no puede tomarse literalmente a partir de dichos períodos de descanso, que pueden interrumpir con el reinicio de sus estudios considerando la garantía del proceso de graduación que la UOC y otras instituciones de España ofrecen a sus estudiantes para facilitar el acceso a una educación con flexibilidad en tiempo de estudios o permanencia (Grau-Valldosera y Minguillón, 2014).

La Universidad de Ciencia y Tecnología de Irán, identifica como factores relacionados con la deserción la baja satisfacción en el desarrollo de los cursos y la falta de control, gestión e interacción con el estudiante, lo que conlleva a promover estrategias orientadas hacia el desarrollo de tendencias motivacionales con un aprendizaje autorregulado y una efectiva interacción de la institución con los estudiantes durante el proceso de aprendizaje (Rostaminezhad et al., 2013). De igual forma, la Universidad de Brasilia en una investigación con estudiantes graduados de programas semipresenciales, evalúa aspectos del ambiente de aprendizaje, demostrando que el uso de entornos virtuales flexibles en tiempo y espacio, costos involucrados, herramientas informáticas y procedimientos de interacción facilitan la permanencia de estudiantes en los programas (Peixoto et al., 2012). La Universidad de Tennessee (Estados Unidos) estudia las variables que influyen en la decisión de persistir o retirarse de un programa de posgrado en línea a través de un ejercicio de percepción con estudiantes, y propone estrategias dirigidas a aspirantes con información requerida para el desarrollo del programa, el apoyo docente y los servicios institucionales (Su y Waugh, 2018).

A partir de una investigación en la que se describen las principales características de la educación a distancia Radovan (2019) analiza modelos enfocados a evitar que los estudiantes abandonen sus estudios, sugiriendo que las circunstancias que afectan la tasa de deserción deben considerarse en la implementación de programas de educación a distancia. Oliveira et al. (2019) hacen lo propio en un estudio adelantado en la Universidad Abierta de Brasil y a través de un análisis de modelado predictivo presentan un enfoque para la identificación temprana de estudiantes en riesgo de abandonar sus estudios en programas a distancia.

Los cursos en línea también han sido objeto de análisis desde la permanencia estudiantil. Lee et al. (2013) examinaron diferencias entre persistencia y abandono de estudiantes mediante cinco factores: apoyo de la familia y el trabajo; control académico, autoeficacia académica, gestión del tiempo y el entorno y habilidades de autorregulación metacognitiva, encontrando que los estudiantes que persistieron contemplan un mayor control académico y autorregulación metacognitiva que los estudiantes que abandonaron los estudios. Por su parte, Aldowah et al. (2020), analizan la afectación de los cursos en línea abiertos masivos a través de un método de toma de decisiones de criterios múltiples que determina como factores centrales: habilidades académicas, experiencia previa, diseño del curso y presencia y apoyo social lo que incide en la falta de compromiso de los aprendices que si bien inician con entusiasmo terminan abandonando sus estudios.

El análisis documental evidencia la evolución que ha tenido el tema de deserción y permanencia estudiantil en la educación superior y su contextualización en diferentes IES, además del interés por la comprensión de los factores determinantes del fenómeno y lo fundamental que se hace la generación de acciones que fomenten la permanencia como desafío de educadores, investigadores y formuladores de políticas.

La presente investigación surge de la necesidad de desarrollar estrategias efectivas que promuevan la permanencia estudiantil en los programas académicos de posgrado de la Universidad de Santander (Colombia) considerando las implicaciones de las dinámicas de deserción y/o permanencia sobre los indicadores de gestión de la institución, especialmente en la Maestría en Gestión de la Tecnología Educativa, programa que lidera a nivel nacional dado el número significativo de estudiantes y su metodología virtual. Con el objetivo de identificar estrategias de permanencia estudiantil a partir de situaciones reales y escenarios deseables para el caso de estudio seleccionado, se espera contribuir al análisis de una problemática que afecta tanto a los estudiantes y sus familias al no concluir con sus proyectos de formación, como a las instituciones al no cumplir con las políticas de cobertura y calidad de la educación y/o las políticas de acceso, uso y apropiación de TIC. 


\section{METODOLOGÍA}

La investigación se basa en un caso de estudio que aborda el programa académico Maestría en Gestión de la Tecnología Educativa ofrecido en modalidad virtual por la Universidad de Santander. Se utiliza la metodología cuantitativa descriptiva para el análisis estadístico de información del programa académico y la percepción de los estudiantes sobre factores que motivan la deserción o permanencia estudiantil. El proceso metodológico se estructura en tres etapas desarrolladas de manera complementaria, las cuales se describen en la Figura 1.

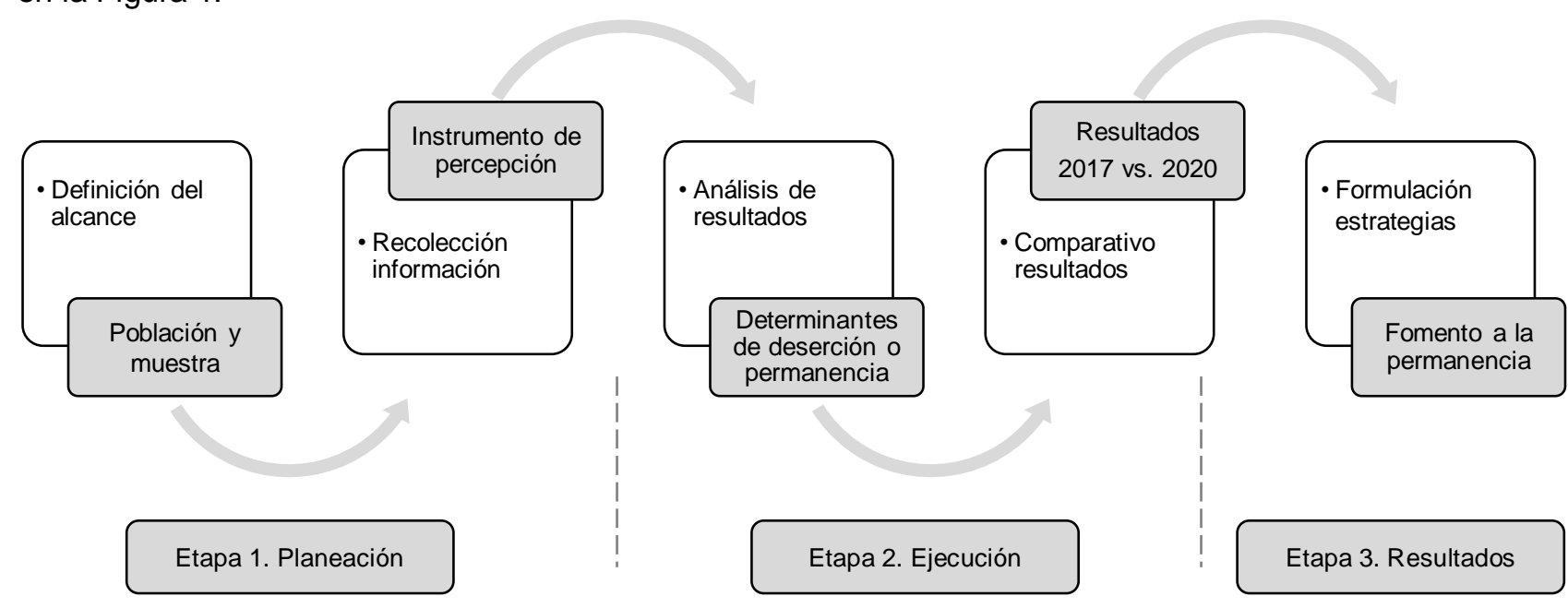

Fig. 1: Metodología de análisis de la permanencia estudiantil en el programa académico

En la etapa de planeación, se precisa la población a intervenir y se determina la muestra del programa académico según cada momento de percepción. Adicional, se define el instrumento de recolección de datos. Durante la etapa de ejecución, se recopilan y analizan respuestas obtenidas a través del instrumento aplicado y se comparan los resultados de los dos momentos de percepción llevados a cabo en 2017 y 2020 , con el fin de corroborar el sentir de la comunidad estudiantil respecto de las motivaciones que influyen en la decisión de abandonar sus estudios o permanecer en el programa académico. Finalmente, en la etapa de resultados, considerando los hallazgos encontrados durante la ejecución se formulan estrategias para el fomento de la permanencia estudiantil en el programa académico, que puedan favorecer la finalización del proceso formativo y la graduación de estudiantes.

Para establecer los motivos que afectan la permanencia de estudiantes en el programa de Maestría en Gestión de la Tecnología Educativa, se elabora un instrumento de percepción considerando los determinantes de deserción sugeridos por el Ministerio de Educación Nacional y un factor adicional denominado "Factor Tecnológico", dada la incidencia que reviste el uso de herramientas y recursos tecnológicos en el desarrollo de programas académicos con metodología virtual. La relación de factores y variables de permanencia en el programa académico, se obtiene del análisis de literatura relacionada con el tema, las novedades académicas de retiro definitivo del programa y suspensión de plan de estudios solicitadas por los estudiantes y el aporte de profesores y personal directivo y administrativo quienes en el marco de su gestión y contacto permanente con estudiantes conocen determinadas situaciones. La Figura 2 muestra la relación de factores y variables determinantes de la permanencia estudiantil en el programa de Maestría en Gestión de la Tecnología Educativa.

El instrumento se aplica en dos momentos de percepción a poblaciones distintas de estudiantes de los cuatro semestres del programa académico con cursos en ejecución en los años 2017 y 2020. La población participante es diferente en cada momento dado que los estudiantes activos en el 2017 cambian su estado académico para el 2020 por graduado, suspendido o retirado del programa. En el primer momento de percepción aplicado en 2017 respondieron el instrumento 1.506 estudiantes, y en el segundo momento en el año 2020, la población que respondió fue de 1.088 estudiantes.

La información recolectada ha sido procesada aplicando técnicas de análisis de datos estadísticos mediante correlación de variables según cada factor determinante de la permanencia, los aspectos de cada factor y la audiencia correspondiente, para ello se han estratificado los datos según las opciones de respuesta seleccionadas. El instrumento aplicado en la recolección de datos tiene un coeficiente de fidelidad del 0,78 según análisis de consistencia de Alfa de Cronbach. 


\begin{tabular}{|c|c|c|c|c|}
\hline Individuales & Socioeconómicos & Académicos & Tecnológicos & Institucionales \\
\hline $\begin{array}{l}\text { - Disponibilidad de } \\
\text { tiempo para } \\
\text { estudiar } \\
\text { - Condiciones de } \\
\text { salud } \\
\text { - Condiciones } \\
\text { Personales } \\
\text { - Cambios en su } \\
\text { motivación para } \\
\text { continuar con los } \\
\text { estudios }\end{array}$ & $\begin{array}{l}\text { - Limitaciones } \\
\text { económicas para } \\
\text { cumplir con los } \\
\text { costos de matrícula } \\
\text { y pecuniarios } \\
\text { - Condiciones } \\
\text { laborales y } \\
\text { ocupacionales } \\
\text { - Condiciones } \\
\text { sociopolíticas } \\
\text { - Relaciones } \\
\text { interpersonales con } \\
\text { dificultades hacia a } \\
\text { profesores y/o } \\
\text { estudiantes. } \\
\text { - Condiciones } \\
\text { climáticas }\end{array}$ & $\begin{array}{l}\text { - Conocimientos } \\
\text { previos sobre las } \\
\text { temáticas del } \\
\text { programa } \\
\text { - Dificultad en el } \\
\text { desarrollo y } \\
\text { evaluación de las } \\
\text { actividades de } \\
\text { aprendizaje. } \\
\text { - Cumplimiento de } \\
\text { requisitos } \\
\text { académicos } \\
\text { - Plan de Estudio } \\
\text { - Otras opciones de } \\
\text { estudio } \\
\text { - Dificultad para } \\
\text { aplicar aprendizajes } \\
\text { en su contexto } \\
\text { laboral y profesional }\end{array}$ & $\begin{array}{l}\text { - Dificultad en conexión } \\
\text { a Internet } \\
\text { - Dificultad de acceso a } \\
\text { herramientas } \\
\text { tecnológicas } \\
\text { - Manejo de plataforma } \\
\text { y/o herramientas } \\
\text { virtuales } \\
\text { - Dificultad en uso de } \\
\text { tecnología para } \\
\text { aplicación en su } \\
\text { contexto laboral o } \\
\text { profesional. } \\
\text { - Disponibilidad y } \\
\text { funcionalidad a } \\
\text { plataforma virtual y } \\
\text { herramientas } \\
\text { tecnológicas. } \\
\text { - Soporte técnico a } \\
\text { usuarios }\end{array}$ & $\begin{array}{l}\text { - Procesos } \\
\text { administrativos: } \\
\text { atención a } \\
\text { estudiantes, } \\
\text { certificaciones, } \\
\text { otros } \\
\text { - Seguimiento y } \\
\text { acompañamiento } \\
\text { de profesores } \\
\text { - Recursos } \\
\text { bibliográficos } \\
\text { - Canales de } \\
\text { atención a usuarios }\end{array}$ \\
\hline
\end{tabular}

Fig. 2: Relación de factores y variables de permanencia estudiantil en el programa académico

\section{RESULTADOS Y DISCUSIÓN}

Para el caso de estudio del programa Maestría en la Gestión de la Tecnología Educativa de la Universidad de Santander, los resultados del primer momento de percepción muestran que los factores con mayor incidencia en la permanencia estudiantil son de carácter individual, socioeconómico y académico, mientras que los factores tecnológicos e institucionales se consideran de menor relevancia. Los resultados del segundo momento, reflejan como factores más influyentes los de carácter individual, institucional y tecnológico, sobre los factores académicos y socioeconómicos. Cualquiera de los escenarios de análisis propuestos por los dos momentos de percepción, permiten a la institución enfocar sus esfuerzos en la formulación de estrategias que contrarresten los diversos aspectos que afectan la continuidad de la formación de los estudiantes.

El comparativo de resultados, muestra además la prevalencia de los siguientes aspectos desde cada factor: 1) Individuales: disponibilidad de tiempo para estudiar. 2) Socioeconómicos: limitaciones económicas para cumplir con los costos pecuniarios. 3) Académicos: cumplimiento de requisitos académicos. 4) Tecnológicos: dificultad en la conexión a internet. 5) Institucionales: seguimiento y acompañamiento de profesores. La Figura 3 presenta los resultados obtenidos en 2017 y 2020 con respecto a los factores que afectan la permanencia de estudiantes en la Maestría en Gestión de la Tecnología Educativa.

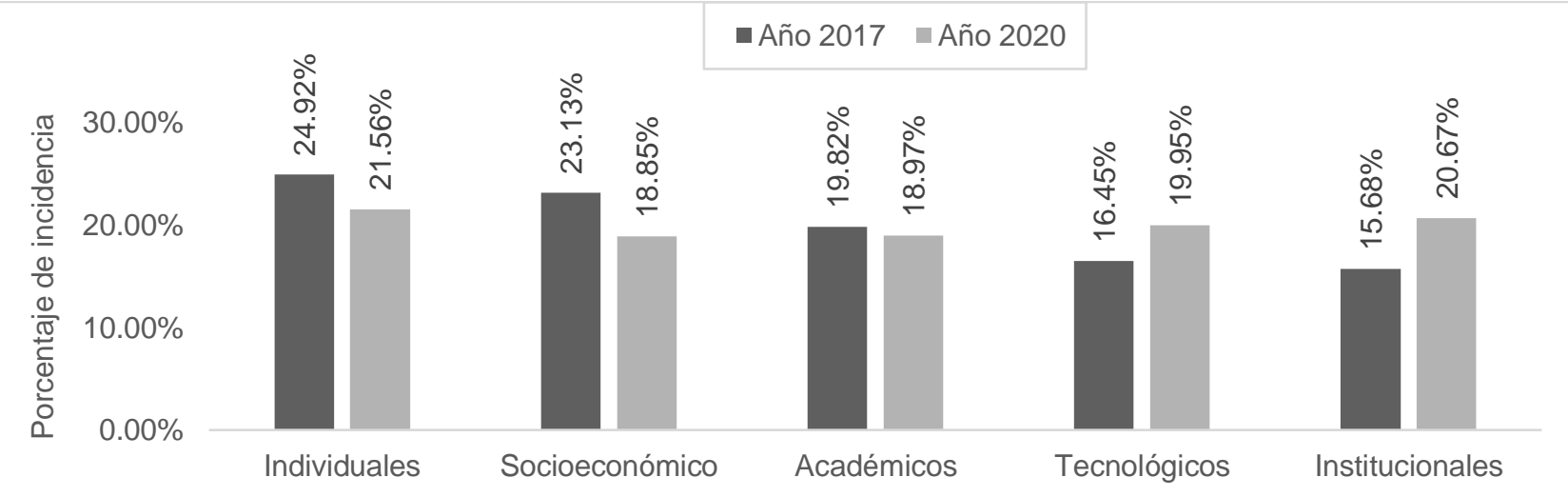

Factores de permanencia

Fig 3: Factores que afectan la permanencia estudiantil en el programa académico

El análisis de resultados a la luz de la revisión de enfoques teóricos y modelos sobre retención de estudiantes de educación superior, justifica el interés por identificar con precisión las variables que afectan la deserción y/o permanencia de estudiantes, pasando de concepciones que atribuían la deserción a la responsabilidad y fallas exclusivas del estudiante, a asumir un espectro más amplio donde las instituciones desde una mirada interna en su gestión también son responsables de cierta forma sobre las decisiones de abandono o permanencia de sus estudiantes, así como la incidencia de factores externos ajenos a la Institución que afectan directamente al estudiantado. 
Desde esta interpretación, se proponen estrategias para fomentar la permanencia y graduación de los estudiantes, enfocadas en los siguientes ejes de trabajo: 1) Atención a Estudiantes: Caracterización de estudiantes; Consejería estudiantil; Bienestar Institucional; Ayudas didácticas; Comunicaciones institucionales; Flexibilización académica y financiera. 2) Gestión Académica: Inducción a estudiantes. Identificación de presaberes; Banco de actividades académicas; Actualización de contenidos; Acceso a recursos bibliográficos; Seguimiento a deserción y permanencia estudiantil. 3) Mejoramiento de la Gestión Docente: Protocolo encuentros sincrónicos; Instrumentos de desarrollo, realimentación y valoración de actividades de aprendizaje; Repositorio trabajos de grado; Espacios de interacción para profesores y estudiantes; Inducción y formación docente; Fomento a la permanencia estudiantil. 4) Fortalecimiento de Sistemas de Información y Comunicación: Sistemas CRM, Alertas Tempranas y Administración Académica; Sistematización Canal videotutoriales; Sistematización rúbricas de evaluación de actividades de aprendizaje. La Figura 4 representa situaciones reales del estudiante que afectan su permanencia en el programa académico y posibles soluciones deseables para la culminación exitosa de su formación.

Las estrategias planteadas para la Maestría en Gestión de la Tecnología Educativa, surgen del aporte del estudio realizado con información académica del programa, en sintonía con lineamientos del MEN (Parody et al, 2015) y buenas prácticas y estudios adelantados en diversas IES. Los resultados de la investigación están en línea con autores como Rostaminezhad et al. (2013), Castro et al. (2020) y Rueda et al. (2020), desde la identificación de diversos factores determinantes de deserción y/o permanencia y la creación de acciones para contrarrestarlos o potencializarlos según corresponda, instando a las instituciones educativas a una mirada focalizada que, lejos de estrategias generales, apunten a una mejor comprensión de las realidades de la comunidad educativa. Grau-Valldosera y Minguillón (2014), Su y Waugh (2018), Lee et al. (2013) y Aldowah et al. (2020) también resaltan la particularización de acciones en contexto, considerando el nivel de formación y la metodología de los programas académicos. La Tabla 1 presenta detalle de los aportes de los autores a los resultados del presente estudio.

Tabla 1: Aportes de la literatura a los resultados de la investigación

\begin{tabular}{|c|c|c|}
\hline & Autores & Aportes \\
\hline 1 & Parody et al. (2015) & $\begin{array}{l}\text { Las recomendaciones del MEN articulan aspectos como el entorno familiar del } \\
\text { estudiante, el ambiente institucional, la integración académica y social, la gestión } \\
\text { docente y calidad de la enseñanza y la satisfacción con el sistema académico. }\end{array}$ \\
\hline 2 & $\begin{array}{l}\text { Rostaminezhad et al. } \\
\text { (2013) }\end{array}$ & $\begin{array}{l}\text { La satisfacción en el desarrollo de la gestión académica y los procesos de } \\
\text { interacción son considerados factores de relevancia en la permanencia de } \\
\text { estudiantes en programas académicos de posgrado e-learning, a partir de los } \\
\text { cuales se promueve la generación de estrategias que reduzcan o dinamicen su } \\
\text { impacto. }\end{array}$ \\
\hline 3 & Castro et al. (2020) & $\begin{array}{l}\text { La relación entre factores demográficos, socioeconómicos y académicos de ingreso } \\
\text { a la universidad con el resultado final de los estudiantes, estimula el seguimiento y } \\
\text { control al desempeño académico exigiendo eficientes sistemas de información } \\
\text { institucional. }\end{array}$ \\
\hline 4 & Rueda et al. (2020) & $\begin{array}{l}\text { Los perfiles de riesgo de deserción destacan factores familiares, socioeconómicos, } \\
\text { institucionales, académicos e individuales, a partir de los cuales se insta a la } \\
\text { creación de programas de acompañamiento y orientación a estudiantes que } \\
\text { intervengan el fenómeno desde diversas perspectivas. }\end{array}$ \\
\hline 5 & $\begin{array}{l}\text { Grau-Valldosera y } \\
\text { Minguillón (2014) }\end{array}$ & $\begin{array}{l}\text { Se destaca la importancia de considerar los contextos particulares de cada } \\
\text { institución educativa a la hora de definir conceptos como permanencia, abandono, } \\
\text { suspensión, readmisión, considerando la relevancia de la condición de flexibilidad } \\
\text { ofrecida a estudiantes de educación superior en línea / a distancia. }\end{array}$ \\
\hline 6 & Su y Waugh (2018) & $\begin{array}{l}\text { Estudios de percepción de estudiantes, desarrollan modelos de análisis útiles para } \\
\text { identificar rangos de variables críticas que influyen en la decisión de persistir o } \\
\text { retirarse de un programa académico atendiendo a circunstancias particulares. }\end{array}$ \\
\hline 7 & Lee et al. (2013) & $\begin{array}{l}\text { El análisis de la persistencia y el abandono estudiantil en programas de formación } \\
\text { en línea, a través de la identificación de factores incidentes, propician el desarrollo } \\
\text { de acciones que fortalezcan la labor institucional desde el bienestar estudiantil y la } \\
\text { gestión académica. }\end{array}$ \\
\hline 8 & Aldowah et al. (2020) & $\begin{array}{l}\text { Los métodos de toma de decisiones de criterios múltiples resultan eficientes en la } \\
\text { identificación de factores que influyen en la deserción de los estudiantes de cursos } \\
\text { en línea abiertos masivos (MOOC). }\end{array}$ \\
\hline
\end{tabular}


La formulación de estrategias para el programa se fundamenta en aspectos internos (debilidades y fortalezas) sobre los cuales se tiene algún grado de control y/o puede influirse directamente, y aspectos externos (oportunidades y amenazas) que favorecen o limitan el éxito de las mismas. La ejecución de las acciones asociadas a dichas estrategias requiere responsables que faciliten el desarrollo de los procesos en los tiempos establecidos, con los recursos asignados y con el nivel de calidad exigido, por tanto, el programa académico debe definir roles y responsabilidades, de forma tal que los involucrados viabilicen la operación y el seguimiento de las acciones planteadas ya sean estas de son de índole preventivo y/o correctivo.

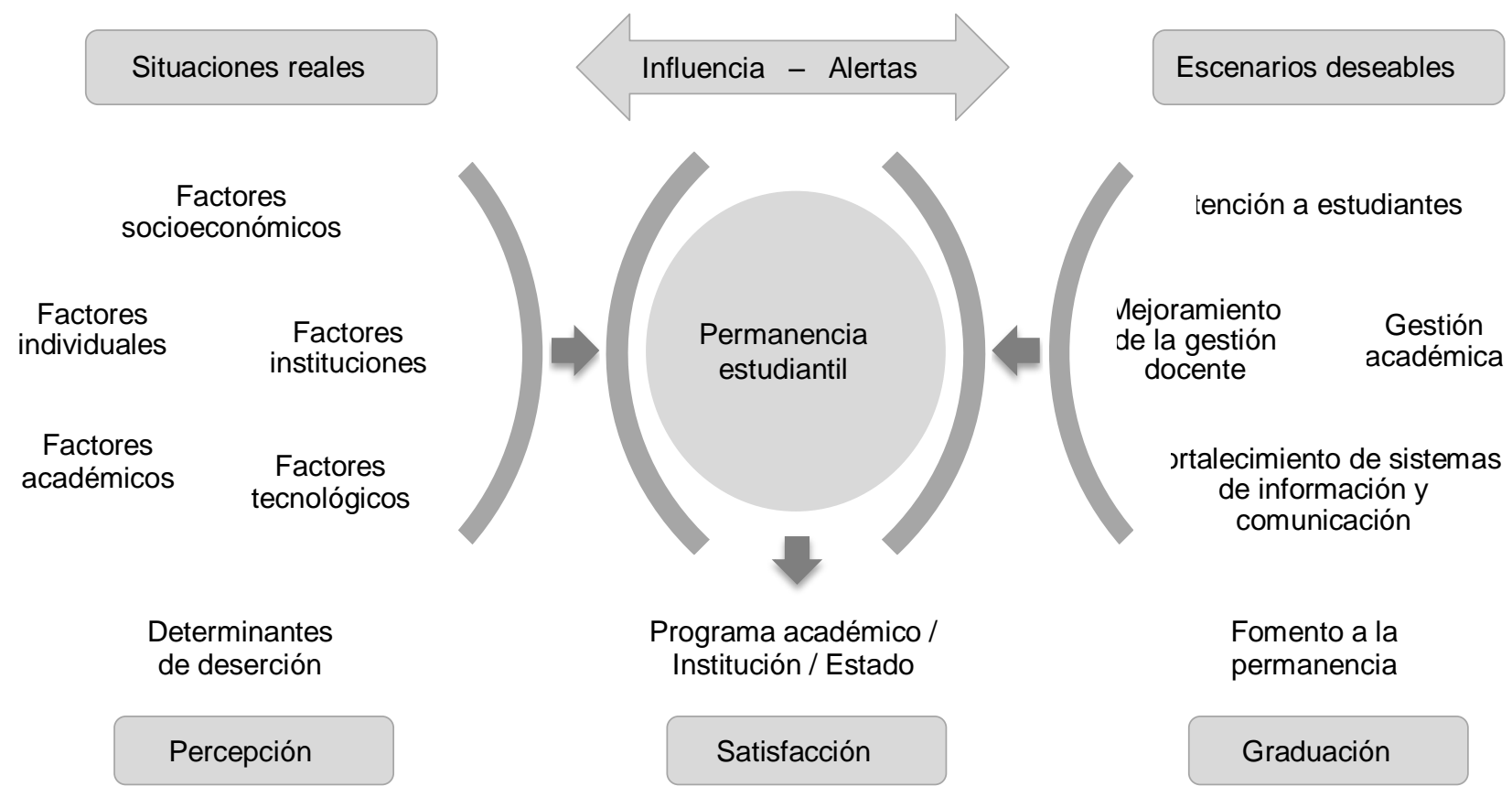

Fig. 4: Dinámica de la permanencia estudiantil en el programa académico

\section{CONCLUSIONES}

El caso de estudio del programa académico "Maestría en Gestión de la Tecnología Educativa" de la Universidad, evidencia que: 1) La diversidad de aspectos que afectan la permanencia de un estudiante en el sistema educativo, hace necesario que el programa y la institución analicen las variables que motivan la deserción de estudiantes y las posibles estrategias que contribuyan en la retención de los mismos. 2) Las estrategias propuestas están enfocadas en fortalecer aspectos de carácter individual, socioeconómico, académico, tecnológico e institucional, que sopesan las posibilidades de permanencia estudiantil en el programa académico, y robustecen la institución en el desarrollo de programas y proyectos. 3) La formulación de estrategias para el programa requiere de la caracterización de la población estudiantil, acciones según el contexto y procedimientos de implementación, seguimiento y control. 4) La permanencia en el programa académico es el resultado positivo de los esfuerzos conjuntos entre institución y estudiante para llevar a cabo el proceso formativo, ambos actores son consecuentes de sus actuaciones, con responsabilidades y compromisos particulares pero complementarios. 5) La responsabilidad de la institución frente a las políticas de cobertura y calidad de la educación y las políticas de acceso, uso y apropiación de TIC, implica esfuerzo, disciplina y mejora continua en las estrategias de fomento a la permanencia y graduación de estudiantes. 6) Las interpretaciones de los hallazgos de este caso de estudio y su aplicabilidad en programas de posgrado e-learning debe determinarse sólo después de una cuidadosa consideración de variables propias del contexto de cada entorno educativo.

\section{AGRADECIMIENTOS}

A la Universidad de Santander, por su disposición frente al desarrollo de la investigación y el acceso a la información requerida.

\section{REFERENCIAS}

Aldowah, H., Al-Samarraie, H., Alzahrani, A.I., y Alalwan, N., Factors affecting student dropout in MOOCs: a cause and effect decision-making model, https://doi.org/10.1007/s12528-019-09241-y, Journal of Computing in Higher Education, 32(2), 429-454 (2020) 
Barragán, S.P., y González, L., Acercamiento a la deserción estudiantil desde la integración social y académica, https://doi.org/10.1016/j.resu.2017.05.004, Rev. de la Educación Superior, 46(183), 63-86 (2017)

Carroll, D., Ng, E. y Bırch, D., Strategies to improve retention of postgraduate business students in distance education courses: an australian case, turkish online Journal of Distance Education, 14(1), 140-153 (2013)

Castro, B.A., Manrique, R.D., González, D., y Segura, A., Trayectoria académica y factores asociados a graduación, deserción y rezago en estudiantes de programas de pregrado de una universidad privada de Medellín (Colombia), http://dx.doi.org/10.4067/S0718-50062020000100043, Formación Universitaria, 13(1), $43-54$ (2020)

Eckert K.B. y Suénaga R., Análisis de deserción-permanencia de estudiantes universitarios utilizando técnicas de clasificación en minería de datos, http://dx.doi.org/10.4067/S0718-50062015000500002, Formación Universitaria, 8(5), 3-12 (2015)

Grau-Valldosera, J., y Minguillón, J., Rethinking dropout in online higher education: the case of the Universitat Oberta de Catalunya, https://doi.org/10.19173/irrodl.v15i1.1628, The International Review of Research in Open and Distributed Learning, 15(1), 290-308 (2014)

Hubackova, S., History and perspectives of elearning, https://doi.org/10.1016/j.sbspro.2015.04.594, Procedia Social and Behavioral Sciences, 191, 1187-1190 (2015)

Huerta, P.C., y Gaete, H.G., Convenios marco y su aplicación en las universidades públicas chilenas: periodo 20152016, http://dx.doi.org/10.4067/S0718-50062019000200105, Formación Universitaria, 12(2), 105-120 (2019)

Lee, Y., Choi, J., y Kim, T., Discriminating factors between completers of and dropouts from online learning courses, http://doi.org/10.1111/j.1467-8535.2012.01306.x, British Journal of Educational Technology, 44(2), 328-337 (2013)

Oliveira, M., Barwaldt, R., Pias, M., y Espindola, D., Understanding the student dropout in distance learning, http://doi.org/10.1109/FIE43999.2019.9028433, 2019 IEEE Frontiers in Education Conference, 1-7 (2019)

Oliveira, P, Oesterreich, S.A. y Almeida, V.L., Evasão na pós-graduação a distância: evidências de um estudo no interior do Brasil, http://dx.doi.org/10.1590/S1678-4634201708165786, Educação e Pesquisa, 44(1), 1-20 (2017)

Paoloni P.V., Martín R.B. y Chiecher A.C., Contextos de aprendizaje en el nivel medio y en la universidad. Percepciones y expectativas de estudiantes que ingresan en carreras de ingeniería, http://dx.doi.org/10.4067/S071850062015000600007, Formación Universitaria, 8(6), 47-56 (2015)

Parody, G., Ariza N., y otros doce autores, Guía para la implementación del modelo de gestión de permanencia y graduación estudiantil en instituciones de educación superior, 1a Ed., 1-229, Editorial Pontificia Universidad Javeriana, Bogotá (2015)

Paura, L. y Arhipova, I., Cause analysis of students' dropout rate in higher education study program, https://doi.org/10.1016/j.sbspro.2013.12.625, Procedia Social and Behavioral Sciences, 109(1), 1282-1286 (2014)

Peixoto, H.M., Peixoto, M.M., y Alves, E.D., Aspects related to the permanence of undergraduate and graduate students in semi-presential classes, https://doi.org/10.1590/S0103-21002012000900008, Acta Paulista de Enfermagem, 25(2), 48-53 (2012)

Piva, F., y De Moraes, J., Student assistance in higher education in Brazil, https://doi.org/10.1016/j.sbspro.2015.01.786, Procedia Social and Behavioral Sciences, 174(1), 1542-1549 (2015)

Popovici, A., y Mironov, C., Students' perception on using elearning technologies, https://doi.org/10.1016/j.sbspro.2015.02.300, Procedia Social and Behavioral Sciences, 180, 1514-1519 (2015)

Radovan, M., Should I stay, or should I go? Revisiting student retention models in distance education, https://doi.org/10.17718/tojde.598211, Turkish Online Journal of Distance Education, 20(3), 29-40 (2019)

Rostaminezhad, M.A., Mozayani, N., Norozi, D., y Iziy, M., Factors related to e-learner dropout: case study of IUST elearning center, https://doi.org/10.1016/j.sbspro.2013.06.100, Procedia Social and Behavioral Sciences, 83, 522-527 (2013)

Rueda, S.M., Urrego, D., y otros tres autores, Perfiles de riesgo de deserción en estudiantes de las sedes de una universidad colombiana, https://doi.org/10.18800/psico.202001.011, Revista de Psicología, 38(1), 275-297 (2020)

Su, J., y Waugh M.L., Online student persistence or attrition: observations related to expectations, preferences, and outcomes, Journal of Interactive Online Learning, 16(1), 63-79 (2018)

Suárez, N., y Díaz, L.B., Estrés académico, deserción y estrategias de retención de estudiantes en la educación superior, http://dx.doi.org/10.15446/rsap.v17n2.52891, Revista de Salud Pública, 17(2), 300-313 (2015)

Tinto, V., Dropout from higher education: a theoretical synthesis of recent research, https://doi.org/10.3102/00346543045001089, Review of Education Research, 45(1), 89-125 (1975)

Urbina, J.E. y Ovalles, G.A., Abandono y permanencia en la educación superior: una aplicación de la teoría fundamentada, https://doi.org/10.18634/sophiaj.12v.1i.290, Rev. Sophia, 12(1), 27-37 (2016)

Von Hippel, P.T. y Hofflinger, A., The data revolution comes to higher education: identifying students at risk of dropout in Chile, https://doi.org/10.1080/1360080X.2020.1739800, Journal of Higher Education Policy and Management, 1, 1-23 (2017) 